\title{
Estrategias didácticas virtuales acogidas por el docente universitario en tiempos de COVID-19
}

\section{Virtual teaching strategies in times of COVID-19}

Recibido 17/04/2021

Aceptado 01/07/2021

Olinda Ninet Girón Ovalle

Maestría en Docencia Universitaria

Universidad de San Carlos de Guatemala

olindaninet@yahoo.com

https://orcid.org/0000-0002-7168-3027

\section{Referencia}

Girón Ovalle, O. N. (2021). Estrategias didácticas virtuales acogidas por el docente universitario en tiempos de COVID-19. Revista Docencia Universitaria, 2(2), 41-51.

https://doi.org/10.46954/revistadusac.v2i2.30

\section{Resumen}

Este artículo proviene de la investigación que se realizó a raíz del distanciamiento social que provocó la pandemia y con ello el cierre de los establecimientos educativos, situación que indujo a la educación remota de emergencia o virtual, lo cual dio continuidad al proceso de enseñanza aprendizaje. En este sentido, El objetivo de esta investigación fue: Describir las estrategias didácticas virtuales adoptadas por los docentes a raíz del COVID-19 en la Facultad de Ciencias Económicas de la Universidad de San Carlos de Guatemala en el año 2020. Se utilizó un enfoque mixto. Para la fase cualitativa se trabajó un diseño descriptivo no experimental transversal, se tomó como muestra aleatoria simple a docentes y estudiantes de la Facultad, quienes participaron mediante la técnica de una encuesta, y para la fase cualitativa se adecuó un diseño fenomenológico, se eligió una muestra no probabilística con participación voluntaria y quedó constituida por doce coordinadores de curso, quienes participaron en la técnica de entrevista estructurada. 
Palabras clave:

estrategias didácticas, clases virtuales, docente universitario, pandemia.
El hallazgo de la investigación indicó, que, dentro de la planificación, se centraron en los contenidos planificados y el cumplimiento de éstos. Dentro de las estrategias de enseñanza se tomó en cuenta la participación individual, grupal y colaborativa, se planificó la clase y se desarrollaron los temas en presentaciones virtuales, se apoyaron de herramientas tecnológicas, como plataformas educativas y redes sociales. En conclusión, se dio un traslado de los contenidos, de la modalidad presencial, a la modalidad virtual.

\section{Abstract}

This article comes from the research that was carried out as a result of the social distancing that caused the pandemic and with it the closure of educational establishments, a situation that led to emergency or virtual remote education, which gave continuity to the teachinglearning process. In this sense, the objective of this research was: Describe the virtual teaching strategies adopted by teachers as a result of COVID-19 at the Faculty of Economic Sciences of the University of San Carlos de Guatemala in 2020.

A mixed approach was used. For the qualitative phase, a descriptive, non-experimental cross-sectional design was used, teachers and students of the Faculty were taken as a simple random sample, who participated using the technique of a survey, and for the qualitative phase a phenomenological design was adapted, a non-probabilistic sample with voluntary participation and was constituted by twelve course coordinators, who participated in the structured interview technique.

The research finding indicated that, within the planning, they focused on the planned contents and the fulfillment of these. Within the teaching strategies, individual, group and collaborative participation was taken into account, the class was planned and the topics were developed in virtual presentations, they were supported by technological tools, such as educational platforms and social networks. In conclusion, the contents were transferred from the face-to-face mode to the virtual

mode.
Keywords: didactic strategies, virtual classes, university teacher, pandemic. 


\section{Introducción}

El distanciamiento social, que provocó la pandemia SARS-CoV-2, obligó a las universidades a cerrar sus puertas, impactó a un número sin precedente de estudiantes en todo el mundo. Por lo que este súbito cambio de conducta social afectó en un $91 \%$ a la educación mundial (UNESCO, 2020), por lo que los gobiernos e instituciones, pidieron sobrellevar la situación y dar continuidad al proceso de enseñanza aprendizaje durante el confinamiento, de una forma segura.

La educación a distancia y específicamente la educación virtual, adquirió mayor relevancia, situación que nunca se había visto en el ámbito educativo. (Belmonte, 2020). Cambió abrupto obligó a que se innovara y actualizara el sistema educativo conjuntamente con la práctica, tal como sucedió en la Facultad de Ciencias Económicas de la Universidad de San Carlos de Guatemala. La Facultad, se vio forzada a innovar su sistema educativo, ya que por más de 82 años la didáctica educativa se presentaba en una modalidad presencial, sin embargo, la crisis, produjo cambios abruptos, sin que hubiera tiempo suficiente para poder establecer directrices por parte de las autoridades educativas y docentes, situación que empujó a la innovación sin estar preparados ni remotamente, se dio un salto de lo presencial a lo virtual.

Por su parte los docentes y estudiantes de la Facultad, trataron de continuar su labor académica con los materiales didácticos y tecnológicos a su alcance, mantuvieron la metodología y estrategias didácticas presenciales a través de las diferentes aplicaciones que el internet puso a su disposición, tanto redes sociales como plataformas educativas mediante los diferentes dispositivos móviles con acceso a internet, introduciéndose así a la educación remota de emergencia.

Esta fue la primera vez en la historia que se presentó un marasmo mundial en el sector académico durante un tiempo indefinido; la pandemia de coronavirus provocó la mayor paralización educativa, y el cierre de establecimientos educativos en más de 160 países, a mediados de julio más de mil millones de estudiantes fueron afectados, señaló el secretario general de la Organización de Naciones Unidas, Antonio Guterres (ONU, 2020), Por su parte, en la investigación se pudo determinar que, de los 116 docentes encuestados el 84\% respondió que, si utilizaban estrategias didácticas virtuales, enfocándose 
básicamente al uso de herramientas virtuales, Mientras que un $89.70 \%$ de los estudiantes respondió que la educación virtual es de buena a excelente.

Esta investigación pretende contribuir al avance de los conocimientos educativos, enfocándose a fortalecer las estrategias didácticas en la educación virtual, para lograr una educación de calidad, que permita a los estudiantes obtener una cobertura de educación virtual total, en un ambiente que facilite los procesos, un clima favorable y la posibilidad de estudiar casi que en cualquier lugar. Según Sampieri (2014), se tiene la expectativa que, en los estudios científicos se contribuyan a recomendaciones más amplias y escenarios diversos, para la solución de problemas o la toma de decisiones futuras. El objetivo de esta investigación es: Describir las estrategias didácticas virtuales adoptadas por los docentes a raíz del COVID-19 en la Facultad de Ciencias Económicas de la Universidad de San Carlos de Guatemala en el año 2020.

\section{Materiales y métodos}

El marco metodológico presentó un enfoque mixto. Para la fase cuantitativa se trabajó desde un diseño descriptivo no experimental transversal; asimismo se determinó una muestra aleatoria simple y se utilizó la técnica de encuesta por medio de formularios de Google forms, a 116 docentes y 446 estudiantes de la Facultad de ciencias Económicas. Para la fase cualitativa se trabajó un diseño fenomenológico se eligió una muestra no probabilística con participación voluntaria y quedo constituida por doce coordinadores de curso, quienes participaron en la técnica de entrevista estructurada. 


\section{Resultados}

Tabla 1 Estrategias didácticas virtuales adoptadas por los docentes a raíz del COVID-19

\begin{tabular}{|c|c|c|c|}
\hline Categoría & $\begin{array}{l}\text { Sub } \\
\text { categorías }\end{array}$ & $f$ & Evidencia \\
\hline \multirow[t]{2}{*}{$\begin{array}{l}\text { Estrategias de } \\
\text { enseñanza }\end{array}$} & $\begin{array}{l}\text { Planificación y } \\
\text { objetivos }\end{array}$ & 12 & $\begin{array}{l}\text { Se planifican los cursos virtuales, en función de los } \\
\text { objetivos en tiempo y horario en que se disponen. } \\
\text { Para el cumplimiento de los programas de contenido y } \\
\text { evaluación. }\end{array}$ \\
\hline & $\begin{array}{l}\text { Individuales, } \\
\text { grupales y } \\
\text { colaborativas }\end{array}$ & 10 & $\begin{array}{l}\text { Se planifica la clase para todo el grupo con la ayuda } \\
\text { de las técnicas de presentaciones virtuales a través de } \\
\text { diferentes aplicaciones como prezi, PowerPoint, videos } \\
\text { en YouTube, Word, Excel entre otros, con el apoyo } \\
\text { de herramientas de plataformas educativas donde se } \\
\text { trabajan foros y casos de discusión. }\end{array}$ \\
\hline \multirow[t]{2}{*}{$\begin{array}{l}\text { Estrategias } \\
\text { instruccionales y } \\
\text { de entorno }\end{array}$} & Herramientas & 12 & $\begin{array}{l}\text { Plataformas como: Zoom, Meet, Classroom } \\
\text { Redes Sociales: whatsApp, Facebook, } \\
\text { Correo electrónico y aplicaciones digitales como prezi, } \\
\text { lucinchard }\end{array}$ \\
\hline & Modalidad & $\begin{array}{l}12 \\
10\end{array}$ & $\begin{array}{l}\text { Sincrónicas: Foros, conferencias, wikis, casos de } \\
\text { discusión, mesa redonda. } \\
\text { Asincrónicas: hojas de trabajo, mapas, proyectos correos } \\
\text { electrónicos y plataformas educativas y redes sociales }\end{array}$ \\
\hline
\end{tabular}

Fuente: elaboración propia, según datos obtenidos de entrevistas a coordinadores, año 2020

Las estrategias didácticas virtuales más utilizadas por los docentes son: las estrategias de enseñanza y las estrategias instruccionales, dándoles mayor relevancia a las plataformas virtuales, sin embargo, también son importantes para el desarrollo del curso las redes sociales, los correos electrónicos y materiales interactivos cabe mencionar que dentro del material interactivo se enfocaron básicamente en las presentaciones de los temas.

Así mismo la planificación a la que se refiere es básicamente a la adaptación del cambio de lo presencial a lo virtual sin mayores modificaciones. Dentro de las actividades que se realizan dentro de las estrategias están las conferencias de los temas o contenidos del curso, los trabajos prácticos de los temas llamados también hojas de trabajo o laboratorios, investigaciones de los alumnos y exposiciones 
y en un menor porcentaje ensayos, debates y videos. Las modalidades más utilizadas para el proceso de enseñanza aprendizaje se da en las clases virtuales sincrónicas, pues se tiene que cumplir con un horario y contenido programado, no obstante, se utiliza en un menor porcentaje las tareas de forma asincrónica.

Tabla 2 Herramientas digitales utilizadas

\begin{tabular}{lcc}
\hline Herramientas digitales & Frecuencia & $\%$ \\
\hline Google Meet & 68 & 0.300 \\
Zoom & 58 & 0.256 \\
Classroom & 27 & 0.119 \\
WhatsApp & 18 & 0.079 \\
Moodle Rad & 10 & 0.044 \\
Facebook & 10 & 0.044 \\
Correo electrónico & 20 & 0.088 \\
YouTub & 7 & 0.031 \\
Canva & 3 & 0.013 \\
Padlet & 2 & 0.009 \\
Prezi & 3 & 0.013 \\
RAD & 1 & 0.004 \\
\hline
\end{tabular}

Fuente: elaboración propia, según datos obtenidos de encuesta a docentes, año 2020

De los 116 docentes encuestados el 84\% respondió que si utilizaban estrategias didácticas virtuales, especialmente en lo que se refiere a estrategias de entorno e instruccionales, dándole mayor énfasis a las herramientas digitales, como lo son las plataformas educativas y aplicaciones para el desarrollo del proceso de enseñanza aprendizaje, ya que en la virtualidad dependen de estas herramientas, su usabilidad y acceso a las mismas, ya que no existe presupuesto se hace uso de herramientas gratuitas en un $99 \%$. 


\section{Figura 1}

Como califican el aprendizaje en la modalidad virtual, los estudiantes

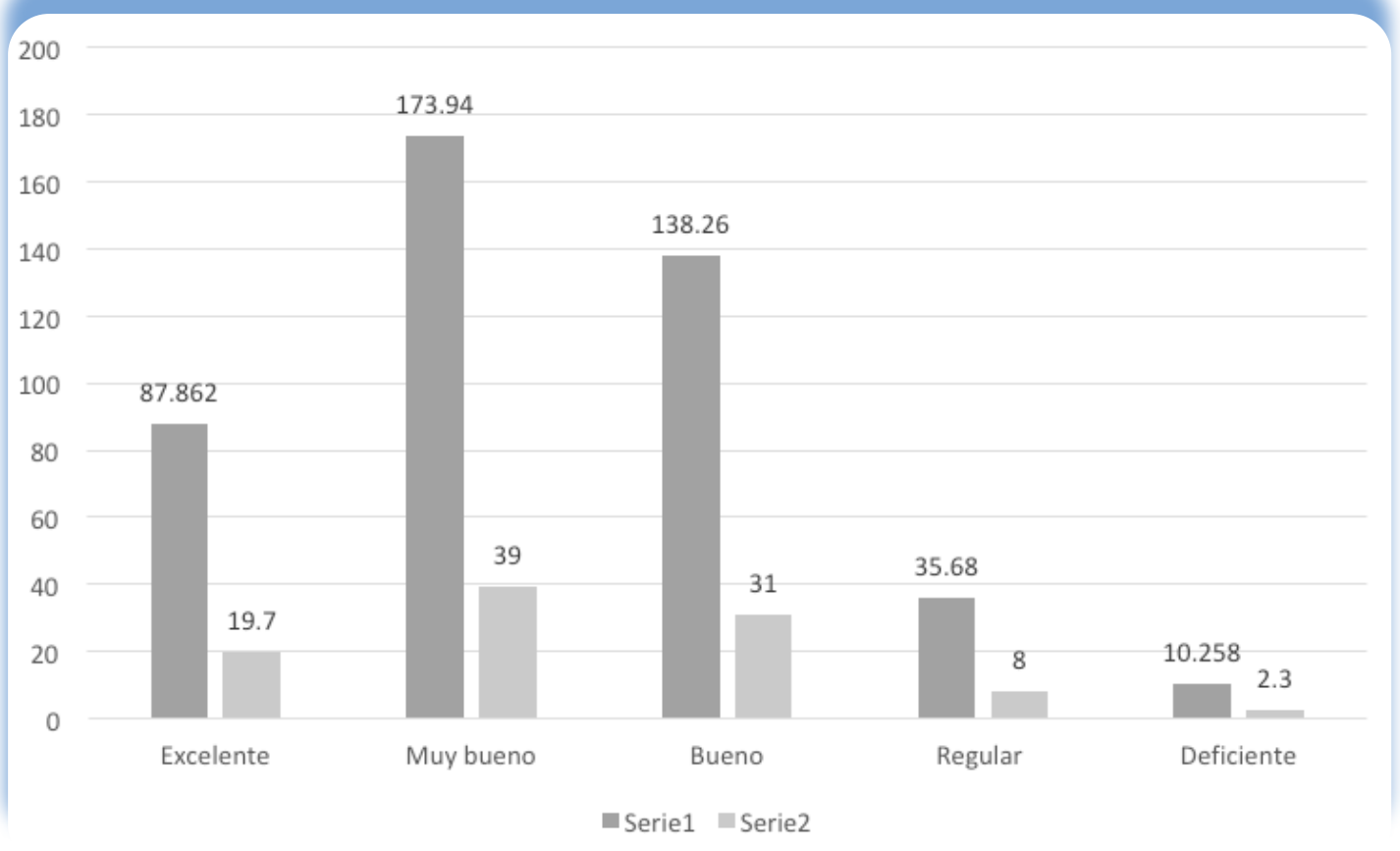

Fuente: elaboración propia, según datos obtenidos de encuesta a estudiantes, año 2020

A pesar de los múltiples inconvenientes que se dieron del traslado presencial al modo virtual los estudiantes se encuentran motivados y participativos en la modalidad virtual, pues como ya se sabe son generaciones nacidas en la tecnología a los cuales no les fue difícil adaptarse. De los 446 estudiantes encuestados únicamente el 10.30 $\%$ de ellos califica el aprendizaje de la modalidad virtual de regular a deficiente. Mientras que un $89.70 \%$ de los estudiantes considera la educación virtual de buena a excelente.

\section{Discusión de resultados}

El autor, Vásquez Rodríguez (2010), señala que las estrategias didácticas, se viabilizan alrededor de los principios, objetivos o propósitos educacionales planteados a nivel de las necesidades de los países o de las instituciones; se evidencia en la conjunción sinérgica y dinámica de la teoría y la práctica que la nutren, la hacen crecer y se fortalecen en la cotidianidad escolar, pues teoría y práctica entran en una relación de complementariedad y contraste, de tal modo que ambas son aspectos 
del conocimiento que capacitan para actuar eficazmente en el terreno de la educación desde cualquier modalidad.

Pues bien, dentro de los resultados de la investigación se pudo determinar, que los coordinadores toman en cuenta, según su planificación y objetivos, a los grupos de participantes por curso y sección, así como el contenido conceptual y procedimental, el factor tiempo, la evaluación en función de los elementos anteriores y principalmente las condiciones espacio-temporales o el ambiente de aprendizaje, que se vio afectado con la emergencia sanitaria.

Por su parte docentes y estudiantes, visualizan las estrategias de enseñanza aprendizaje, desde la modalidad, metodología y actividades didácticas aplicadas, pues dentro de los resultados se pudo observar que no estaban preparados para afrontar una didáctica completamente virtual, sin embargo se adaptaron los contenidos de los programas educativos a la modalidad virtual de forma abrupta; fueron las plataformas educativas gratuitas las herramientas principales para dar continuidad a éste proceso, básicamente se dio un salto de la modalidad presencial a la enseñanza remota de emergencia, como se demuestra en la tabla número uno.

La adaptación de la planificación referente a los objetivos y contenidos curriculares, la didáctica y las medidas tomadas con respecto a la evaluación y calificación de los estudiantes durante la emergencia, ha sido compleja, ya que no se contaba con la experiencia didáctica virtual y las dificultades que esta presenta a la aplicación de las estrategias de enseñanza aprendizaje, especialmente en la evaluación objetiva, pues simplemente se ha dirigido de una forma brusca a la modalidad virtual, señala (Cáceres-Muñoz, 2020).

El hallazgo de la investigación indica, que, dentro de la planificación, según los resultados obtenidos de las entrevistas a coordinadores de curso, y las encuestas mediante cuestionarios de Google Meet planteadas a docentes y estudiantes, se pudo observar que se centran en los contenidos planificados y el cumplimiento de estos mediante la dosificación programada dentro de cada tema y período de tiempo asignado a cada clase, según horario y día que corresponda, así como la programación de dos exámenes parciales y un final. 
Dentro de las estrategias de enseñanza se toman en cuenta la participación individual, grupal y colaborativa, se planifica la clase para todo el grupo o sección asignado, desarrollo de temas en presentaciones virtuales a través de diferentes aplicaciones como Prezi, PowerPoint, videos en YouTube, Word, Excel entre otros, para la disertación del tema, se apoyan de herramientas como plataformas educativas dentro de las cuales las más utilizadas son las de uso gratuito, dentro de estas se pueden mencionar: Zoom, Meet, Classroom y Edmodo, también se utilizaron redes sociales tales como WhatsApp, Blogstop y Facebook, medios donde se trabajan foros, exposiciones y casos de discusión de forma sincrónica, como si fuera una clase presencial, realizan tareas como laboratorios, investigaciones, hojas de trabajo, mapas, ensayos entre otros, para trabajar de forma asincrónicas. Cabe mencionar que en todo este proceso se respeta la libertad de criterio docente.

Sáez y colaboradores (2020) afirman que, sin contar con recursos, formación sobre métodos y estrategias pedagógicas de educación a distancia y/o virtuales, o ninguna plataforma de aprendizaje definida, además, sin instrucciones claras por parte de la administración, se inicia una educación virtual. La finalidad primordial es dar continuidad con las actividades pedagógicas a través de plataformas virtuales, para generar conocimiento desde fuera de las instalaciones universitarias, no obstante dándole seguimiento al proceso de enseñanza aprendizaje, para contribuir al acompañamiento de los saberes en los estudiantes para el logro de las competencias planificadas, de una forma segura desde cada uno de sus hogares.

Se pude concluir, según resultados, que tomaron en cuenta algunas estrategias de entorno; por la situación social en la que se vive, enfocándose principalmente en herramientas tecnológicas tanto dispositivos como aplicaciones, otras estrategias instruccionales, actividades tales como hojas de trabajo, exposiciones, mapas mentales, ensayos en menor proporción según la planificación presencial, sin embargo la frecuencia con que lo realizan no es constante, con lo cual se compromete el tipo de aprendizaje que se realiza, las estrategias de enseñanza se realizan de forma inconsistente sobre todo cuando se trata de estrategias específicas, dado a que por la emergencia no se tubo ninguna preparación en la educación virtual, trasladándose automáticamente a una educación remota de emergencia. 
Por lo mencionado anteriormente, se recomienda, dar capacitación constante a los docentes en la nueva modalidad y a su vez lograr que los docentes se involucren en el conocimiento de las estrategias didácticas virtuales y poderlas identificar desde sus diferentes modalidades, desde las estrategias de enseñanza, aprendizaje, instruccionales, de entorno y evaluación para aprovechar todas sus ventajas y lograr un aprendizaje significativo y autónomo en los estudiantes.

\section{Agradecimientos}

Agradezco a la Facultad de Ciencias Económicas de la Universidad de San Carlos de Guatemala, por brindarme la oportunidad de realizar la investigación de estrategias didácticas virtuales bajo la colaboración de sus autoridades, coordinadores, docentes, estudiantes, y personal administrativo, que contribuyeron a los resultados de la presente investigación. Así mismo agradezco la colaboración al maestro Edwing García por la revisión de este artículo, a la Dra. Walda Paola María Flores Luin como promotora del desarrollo de este articulo y docente de curso de tesis.

\section{Referencias}

Cáceres-Muñoz, J. (17 de junio de 2020). Revista Internacional de Educación para la Justicia Social, 2020. file:///C:/ Users/ASUS/Downloads/12603-Texto\%20del\% 20 art\%C3\%ADculo-34025-1-10-20200723.pdf

Belmonte, C. M. (29 de abril de 2020). Pandemia: maestros, tecnología y desigualdad https://educacion.nexos.com.mx/?p=22860NU. (28 de agosto de 2020). https://www.coronavirusgripe. com/articulo/noticias/onu-advierte-desastre-educacioncovid-19/20200805081314004747.html

Sáez, F. T., Navas, M. F., Rodríguez, R. M., Robles, A. S., Romero, F. J., \& Fuentes, A. Y. (12 de junio de 2020). Revista Internacional de Educación para la Justicia Social, 2020. file:///C:/Users/ASUS/Downloads/12603-Texto\%20del\%20 art\%C3\%ADculo-34025-1-10-20200723.pdf 
Sampieri, R. H. (2014). Metodología de la Investigación sexta edición.

En R. H. Sampieri, Metodología de la Investigación sexta edición (pág. 634). Mexico: Mc Graw Hill Education.

UNESCO. (2020). https://es.unesco.org/covid19/educationresponse

Vásquez Rodríguez, F. (2010). Estrategias de enseñanza,CLACSO. http://biblioteca.clacso.edu.ar/Colombia/fce-unisalle/20170117011106/ Estrategias.pdf

\section{Sobre la autora}

Es licenciada en Administración de Empresas, en la Facultad de Ciencias Económicas, de la Universidad de San Carlos de Guatemala, docente supervisora de tesis, en el área de EPS de la Facultad de Ciencias Económicas, y actualmente cursa la maestría en Docencia Universitaria de la Facultad de Humanidades de la Universidad de San Carlos de Guatemala.

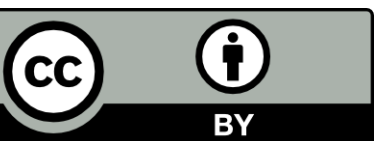

Este texto está protegido por una licencia Creative Commons 4.0.

Usted es libre para compartir, copiar y redistribuir el material en cualquier medio o formato y adaptar el documento, remezclar, transformar y crear a partir del material para cualquier propósito, incluso comercialmente, siempre que cumpla la condición de atribución: usted debe reconocer el crédito de una obra de manera adecuada, proporcionar un enlace a la licencia, e indicar si se han realizado cambios. Puede hacerlo en cualquier forma razonable, pero no de forma tal que sugiera que tiene el apoyo del licenciante o lo recibe por el uso que hace. 